\section{Отиссье Д.}

д-р управленческих наук (HDR), директор Департамента организационных изменений Бизнесшколы ESSEC, Сержи; профрессор Школы Менеджмента IAE Gustave Eiffel Университета Paris-Est Créteil, Кретей, Франция

e-mail: autissier@u-pec.fr

Пащенко - де Превиль Е.В.

д-р психол. наук (PhD), научный сотрудник Департамента организационных изменений Бизнесшколы ESSEC, Сержи, Франция; ассоциированный науч. сотрудник Института психологии РАН, Москва e-mail: elena@depreville.net

\section{Рэнар T.}

ведущий специалист по использованию коллективного интеллекта - Группа сплоченности человеческих ресурсов, HR-департамент, Национальное общество французских железных дорог (SNCF), Париж, Франция

e-mail: thierry.raynard@sncf.fr

\section{Autisier David}

Doctor of Management (HDR), Director of the ESSEC Chair of Change Management, Cergy; Professor of Management School IAE, Gustave Eiffel,

University Paris-Est Créteil,

Créteil, France

e-mail: autissier@u-pec.fr

Pachtchenko-de Préville E.V.

Doctor of Psychology (PhD),

Researcher of the ESSEC Chair of Change Management, Cergy, France. Associated Researcher of Institute of Psychology, Russian Academy of Sciences, Moscow e-mail: elena@depreville.net

\section{Raynard Thierry}

Leading Expert on Collective Intelligence, Human Resources Cohesion Group, HR-Department, National Society of French Railways (SNCF), Paris, France

e-mail: thierry.raynard@sncf.fr

\section{Обучающие сети - альтернатива традиционным методам управления организационными изменениями. Из опыта Национального общества французских железных дорог}

\begin{abstract}
Аннотация
В условиях современной нестабильной экономической обстановки организации, предприятия и компании должны постоянно адаптироваться и вводить инновации, чтобы оставаться конкурентоспособными. Для стимулирования инноваций и усовершенствования адаптационных механизмов организация (компания) должна способствовать раскрытию потенциала своих сотрудников, предоставляя им свободу действий в рамках их полномочий, другими словами, она должна стать обучающейся. Обучающие сети представляют собой одну из форм обучающейся организации и могут быть использованы в качестве альтернативы традиционным методам управления организационными изменениями, вовлекая в них наибольшее количество участников для обеспечения эсффективного перехода к новому способу функционирования, выгодному одновременно для компании, сотрудников и клиентов. В настоящей статье рассматриваются ключевые принципы обучающих сетей и приводятся основные результаты их использования в Национальном обществе французских железных дорог, где они применяются начиная с 2007 г.
\end{abstract}

Ключевые слова:

обучающаяся организация, обучающие сети, организационные изменения, управление организационными изменениями.

\section{Learning Networks: an Alternative to Traditional Methods of Managing Organizational Change. What We Can Learn from the Experience of the National Society of French Railways}

\begin{abstract}
In an unstable economic environment, organizations and companies must constantly adapt and innovate to remain competitive. To innovate and develop its ability to adapt, the organization (company) must release the power of the thoughts and actions of its employees, it must become a learning organization. Learning networks are one of the forms of the learning organization and an alternative to traditional methods of managing organizational change. Learning networks can be used while driving organizational change and should involve the largest number of participants and introduce change whilst satisfying the needs of customers, employees and the company. In this article, we will present the keys to operating learning networks and some key results achieved through learning networks within the National Society of French Railways, where these networks have been in use since 2007.
\end{abstract}

Keywords:

learning organization, learning networks, organizational change, management of organizational changes.

\section{Введение}

Ускорение изменений окружающей среды, возрастающие требования клиентов и усиливающаяся конкуренция увеличивают сложности функциони- рования организаций, компаний и предприятий в целом и производственных отношений в частности.

Реагировать на эту все возрастающую сложность $[8,9]$ как внешней, так и внутренней среды необ- 
ходимо комплексно, т.е. как на экономическом, технологическом, так и на социально-организационном уровнях.

Компании «умные (intelligentes)», по определению Ю. Ландье [6] или «обучающиеся» организации, в терминологии П. Сенге [11], находятся на пути непрерывного совершенствования и формируют у сотрудников общее видение будущего организации, столь необходимое для адаптации к сложной, нестабильной современной экономической обстановке.

По мнению Питера Сенге, автора «Пятой дисциплины» [11], обучающимися являются организации, в которых сотрудники совместно овладевают навыками целостного видения их организации и разрабатывают единую стратегию действий, постоянно расширяют свой потенциал для достижения желаемых ими результатов, отказываются от привычных способов мышления и открыто общаются друг с другом. Увидевшие свет на основе работ Криса Арджириса [2] и Питера Сенге [11-13] теоретические положения и практика обучающейся организации успешно развиваются начиная с 1990 г.

К признакам, присущим обучающейся организации, относятся следующие:

- создание обстановки, в которой сотрудники являются созидателями условий, в которых они работают и которая способствует обучению новым методам работы в реальных ситуациях;

- совместное обучение с целью адаптации, при помощи объединения различных иерархических уровней, без опасения затрагивания различия во взглядах;

- организация работы с учетом знаний и опыта каждого сотрудника и обобщение этих знаний и опыта, т.е. мобилизация коллективного интеллекта;

- позволение сотрудникам самим экспериментировать в сфере их влияния те инновации, авторами которых они явились.

Эта философия de l'apprenance, которой свойственны открытость, умение смотреть на вещи со стороны, принимать во внимание различные точки зрения и т.д., положена в основу обучающих сетей, которые представляют собой одну из форм обучающейся организации и являются одним из рычагов управления организационными изменениями. Apprenance - неологизм французского языка, для которого в русском языке пока, возможно, нет эквивалента. В качестве предварительного перевода можно предложить термин «обучаемость», но понимая его не только и даже не столько на индивидуальном, сколько на коллективном уровне (Прим. авторов). Вместе с тем это понятие выражает жела- ние и способность к обучению на четырех уровнях: индивидуальном, организационном, межорганизационном и социетальном (см. подробнее [14]).

Для многих организаций и компаний управление изменениями имеет первостепенное значение, так как речь идет об их выживании $([5,7]$, упоминаемые в [3]). При этом для внедрения организационных изменений в них продолжают использоваться два традиционных метода: иерархическое и проектное управление, хотя они имеют достаточно много ограничений.

Так, если организация мобилизует управленческую иерархию для проведения организационных изменений, т.е. проводит их методом «сверху вниз», следствием является то, что большая часть сотрудников (адресатов, бенефициаров перемен) оказывается в роли исполнителей, а не в роли актеров и созидателей, имея ограниченные возможности для высказывания. В результате, осознание важности изменений и вероятность модификации поведения (с точки зрения вовлеченности и приверженности) являются ограниченными.

В случае управления изменениями в проектном режиме, проектная группа несет ответственность за развертывание данного процесса для достижения поставленной цели. При этом не учитывается тот факт, что, как это показывают периодически проводимые консалтинговыми компаниями исследования (например, Gartner Group), один проект из двух терпит неудачу, потому что ему не удается заручиться поддержкой и добиться приверженности. Кроме того, метод проектного управления не подходит для осуществления таких изменений, как, например, инновационные, для которых решение не может быть продумано и предложено заранее, без участников самого процесса изменений. Поэтому в настоящее время более эффективным представляется проведение организационных изменений, скорее, способом соконструирования их траектории, чем на основе применения предопределенного плана.

Обучающие сети представляют собой альтернативу двум вышеобозначенным методам организационных изменений, привлекая к совместной работе представителей различных специальностей «вне иерархии», для совместного использования практического опыта. Речь идет об особом качестве отношений, например, если в одной группе обучающей сети находятся бок о бок члены различных иерархических уровней, то мнение каждого имеет одинаковую ценность. Сравнение между проектной группой и группой обучающих сетей предложено в табл. 1. 
Таблица 1

Различия между проектной группой и группой, которая работает в рамках обучающих сетей

\begin{tabular}{|l|l|}
\hline \multicolumn{1}{|c|}{ Проектная группа } & \multicolumn{1}{|c|}{ Группа обучающих сетей } \\
\hline $\begin{array}{l}\text { Аниматором является } \\
\text { руководитель проекта }\end{array}$ & $\begin{array}{l}\text { Аниматор осуществляет } \\
\text { недирективный менеджемент: } \\
\text { все участники равны }\end{array}$ \\
\hline Проект обязателен & $\begin{array}{l}\text { Каждый член группы на } \\
\text { добровольной основе } \\
\text { вкладывает свою лепту в проект }\end{array}$ \\
\hline $\begin{array}{l}\text { Руководитель проекта имеет } \\
\text { заранее предусмотренные } \\
\text { бюджет, график и выходной } \\
\text { продукт }\end{array}$ & $\begin{array}{l}\text { Группа сама выбирает } \\
\text { направление своей деятельности }\end{array}$ \\
\hline $\begin{array}{l}\text { Группа формируется с учетом } \\
\text { экспертности и компетенций } \\
\text { ее членов }\end{array}$ & $\begin{array}{l}\text { Формирование группы } \\
\text { происходит на основании } \\
\text { предпочтений и желания } \\
\text { каждого участника }\end{array}$ \\
\hline
\end{tabular}

Обучающие сети позволяют тем сотрудникам на рабочих местах, которые будут участниками процесса изменений и его непосредственными воплотителями в ходе деятельности, работать бок о бок с инициаторами того или иного изменения над вопросом условий его реализации. Вся иерархическая цепочка управления бывает мобилизованной во время практических встреч в рамках обучающих сетей для обмена опытом и экспериментирования, что способствует укреплению проводимым изменениям. Таким образом, обучающие сети отличаются от иерархического и проектного управлений.

В обучающих сетях их участникам предлагается на добровольной основе участвовать в создании изменений, и именно в процессе совместного конструирования изменений они начинают предлагать решения. При этом процесс выработки решений является не менее важным, чем само решение. Это один из основных принципов обучающего процесса.

Опираясь на опыт, полученный в компании SNCF, сначала рассмотрим, что представляют собой обучающая сеть в целом, чтобы затем остановиться на том, каким образом обучающие сети влияют на увеличение производительности компании. SNCF (Société nationale des chemins de fer français - Национальное общество французских железных дорог) является одной из самых крупных транспортных компаний в Европе. Годовой оборот в 2015 г. составил 32 млрд евро (согласно данным предварительной оценки [10]).

\section{Обучающая сеть. Что это такое?}

В литературе предлагаются различные определения понятия обучающей сети, отличающиеся друг от друга по уровню сложности.

Так, по мнению Ф. Зарифьяна [15], организация в форме сети, отражает потребность компании рабо- тать в трансверсальной манере. Речь идет о привлечении в рамках конкретного проекта различных команд для увеличения общей производительности компании. При этом каждая команда несет полную ответственность за успех на ее участке работы. Главный принцип организации, работающей в сетевой форме, заключается в том, чтобы способствовать взаимодействию между различными командами, профессиями и методами с целью повышения эффективности.

Для того чтобы организация в форме сети успешно функционировала, необходимо соблюдение следующих условий:

- поставленные цели должны быть не слишком абстрактными и достижимыми, учитывали возможности команд;

- необходимо пристально отслеживать случаи возникновения напряженности и разногласий, которые могут возникать из-за того, что состав участников сети очень разнообразен.

Мы предлагаем следующее определение обучающим сетям: обучающие сети - это возможность учиться вместе и в процессе деятельности, вокруг ее объекта, для того, чтобы совместно конструировать изменения в пределах сферы своего влияния перед тем, как их внедрять.

Обучающие сети позволяют сотрудникам, в них участвующим, расширять их собственные горизонты и совместно конструировать представление о будущем компании. Этот метод позволяет всем заинтересованным сторонам обсуждать существующую ситуацию, поставленную цель и способы ее воплощения в устойчивую практическую реальность. Он основан на использовании коллективного обучения, базирующегося на желании каждого учиться и принимать на различных уровнях ответственность. Для функционирования обучающих сетей необходимо создавать специальные места, т.е. пространство для работы над устранением барьеров в процессе взаимодействия, обеспечивая постоянное взаимное приспосабливание участников.

\section{Когда использование обучающих сетей более уместно, чем других методов менеджмента, например, иерархического или проектного управления?}

Ответ на вопрос зависит, с одной стороны, от ожидаемого уровня обучения, а с другой - от степени сложности рассматриваемого вопроса (проблемы).

\section{Ожидаемый уровень обучения}

Обучающая сеть является своего рода лабораторией, где участники ставят под сомнение свои убеждения и представления, при этом обеспечивая кон- 
кретные и оперативные решения. В зависимости от сценария трактовки той или иной проблемы, степени ее сложности, состава участников и требований, диктуемых результатом, обучающие сети обеспечивают понимание проблемного вопроса в процессе деятельности и на основе того представления о ней, которое создается самими участниками.

Обучающая сеть, как правило, мобилизуется не только для изменения практик, но и для изменения представлений и норм. Действительно, со ссылкой на работы К. Аржириса (См. [1]), уместно предположить, что изменения так называемого второго уровня являются труднопроизводимыми в условиях иерархических отношений, где преобладает асимметрия власти, так же, как и при использовании проектного управления, предусматривающего получение заранее запрограммированного результата.

\section{Сложность рассматриваемого вопроса (проблемы)}

Проблема может быть отнесена к комплексным или к сложным, когда среда для деятельности по ее разрешению позволяет множество комбинаций. В среде такого рода трудно точно знать, что и когда произойдет, так как она находится в процессе развития. Это приводит к сложности выделения бинарных причинно-следственных связей и диктует необходимость системного подхода. В таком контексте, менеджмент все менее осуществляет функции контроля, а его роль все более сводится к умению генерировать продвижение и использовать позитивную динамику для получения ожидаемых результатов. Лидером становится не тот, кто имеет доступ к информации или занимает командную позицию, а тот, кто имеет легитимность для создания и капитализации человеческой динамики. В условиях комплексной ситуации важно, чтобы сотрудники могли обсуждать окружающие их факторы, обмениваться опытом и представлениями для выработки общего понимания проблемы и связанных с ней плана действий; именно это и происходит в процессе работы обучающих сетей.

\section{Тематики обучающих сетей}

Анализ опыта обучающих сетей в компании SNCF позволяет выделить пять совокупностей тем, чаще востребуемых и затрагиваемых, чем при использовании других методов управления. Инновации являются центральным элементом этих тематик.

\section{Инновации}

Так же, как и при проведении мозгового штурма (brainstorming), в обучающих сетях речь идет о нахождении новых идей для новой продукции и (или) новых способов деятельности. Процесс инноваций может быть символически выражен через образ воронки: надо постепенно и с помощью различных механизмов подвести участников к пересмотру их способов мышления, для того чтобы интегрировать новое видение и в конечном счете переосмыслить элементы их повседневного окружения.

\section{Тема для предварительного изучения}

Выбор темы для предварительного изучения имеет целью дать участникам возможность понять новую концепцию, не имея для этого достаточного опыта и знаний.

Например, руководителям было предложено начать использовать «экологический менеджмент». Это понятие было недостаточно известным и мало используемым как в самой компании, так и за ее пределами. Надо было изучить его, чтобы сформировать предварительное представление о его признаках и свойствах, особенностях анализа и операционализации.

\section{Решение проблемы}

Определенная и оцениваемая как достаточно важная проблема может быть поставлена перед коллективом для разрешения в условиях жестких ограничений, например, временных. В этом случае задача, стоящая перед обучающей сетью, будет заключаться в точном определении причин данной проблемы, в извещении о них других и подготовке плана действий. При этом процесс размышления над проблемой направлен как на осознание ее важности, так и на предложение реальных и оперативных действий.

Например, последовательная потеря нескольких тендеров может привести основных заинтересованных сторон компании к мобилизации обучающей сети для выявления причин и определения корректирующих действий.

\section{Диагностика ситуации}

Проблемная ситуация, или ситуация по которой возникают вопросы, может стать предметом диагностики с помощью обучающей сети. Под «диагностикой» здесь мы понимаем формализацию объяснительных элементов функционирования или выявление состояния подразделения, проекта, группы, компании или какого-то явления. Акцент в этом случае делается на поиск конкретных фактов о данной ситуации и ее анализ в пространстве и во времени. Достижение объективного понимания лежит в основе всего этого. Во время и после обмена мнениями участники разрабатывают определенный способ интерпретации ситуации, интегрируя раз- 
личные точки зрения группы, и делают более или менее обобщенные выводы.

\section{Привлечение внимания (возбуждение интереса) $\kappa$ теме}

Некоторые темы мало или вообще не известны в организациях. Иногда они известны в общем, но знание о практических и операциональных элементах отсутствует. Возьмем, например, понятие «качество жизни на работе» (авторский перевод французского выражения la qualité de vie au travail). Хотя каждый может иметь представление о чем идет речь, тем не менее основные заинтересованные лица не всегда осведомлены о его важности данного понятия и его практических аспектах. Обсуждение этого вопроса имеет целью дать определение этому феномену, выработать объективное оценивание его важности и предоставить элементы для его использования.

Именно такой подход лег в основу работы над темой «между поколениями», предложенной и рассматриваемой в сети HR компании SNCF.

\section{Участники обучающей сети}

Кого приглашать для участия в обучающей сети? Этот вопрос на самом деле сложнее, чем кажется. Нужно ли приглашать всех тех, кто имеет отношение к той или иной теме? Сеть должна состоять только из добровольцев или также из тех, кто положительно к ней относится? Должна ли она объединять представителей нескольких иерархических уровней или не включать руководителей?

Наблюдение практик обучающих сетей SNCF позволяет предложить несколько простых правил отбора участников.

Правило № 1: Найти «спонсора» (руководителя, который будет поддерживать сеть)

Важно, чтобы обучающая сеть имела «спонсора» - из менеджмента, а лучше из топ-менеджмента компании. Поиск так называемого иерархического «прикрытия» не противоречит принципам обучающих сетей. Напротив, это способ заинтересовать и привлечь руководителей для того, чтобы они вкладывали свои силы в развитие обучающей сети. Это также способ получения институционального признания сети, ее членов и результатов. Спонсора, как правило, выбирают в зависимости от его близости к теме, над которой работает обучающая сеть, и ее задачи, с учетом степени его интереса к обучающим сетям и его убеждений в их отношении. Кроме того, его собственные способности к обучению в процессе работы обучающей сети также являются ключевым элементом.
Правило № 2: Идентифицировать тех «без кого не обойтись»

В большинстве случаев работа над тем или иным вопросом по той или иной тематике требует технических знаний, чтобы избежать искажения смысла (неверных толкований) и стерильных дискуссий и обобщений.

Каждая организация имеет своих «ключевых лиц» - будь то экспертов и (или) лидеров. Важно привлечь этих людей, занимающих различные позиции на разных иерархических уровнях, в обучающую сеть. Благодаря их харизме, техническим и межличностным навыкам, а также тонкому пониманию ситуации, они обладают реальными возможностями положительного влияния.

В связи с тем что они очень мобильны и являются своего рода резонаторами в организации, необходимо проявлять осторожность при их включении в обучающую сеть, так как необходимо, чтобы они оставляли в ней достаточно места для других. Они должны оставаться лидерами и в то же время не препятствовать обмену мнениями и затем распространять полученные результаты.

Анализ ряда обучающих сетей SNCF показал, что их в обучающей сети бывает $25 \%$ от общего числа участников.

Правило № 3: Привлечение «производителей пракmuк»

В основе обучающей сети лежит обмен практиками. Именно обмен, альтернативно сочетающий повествование (описание существующего) и предложения (выработку решений), является краеугольным камнем обучающих сетей, способствуя лучшему пониманию как собственной практики, так и практик другого человека. В обучающих сетях знание есть не что иное, как результат обмена между практикующими, т.е. «производителями практик». Хорошего «производителя», кроме опыта, отличает качество его участия в сети, которое характеризуется благожелательностью, объективностью и умением посмотреть на себя со стороны. В самом деле, одного опыта недостаточно, важно также демонстрировать активное участие и проявлять сотрудничество наряду с сильным желанием менять положение дел.

Некоторые участники являются «производителями» вдвойне, в силу их собственной практики в компании того, что они поддерживают хорошее функционирование обучающей сети и организуют процесс обсуждения проблем. С этой точки зрения они самая важная категория участников, так как являются ее координаторами и 
руководителями дискуссий, т.е. аниматорами, хотя все участники обучающей сети в какой-то степени являются таковыми благодаря их активному взаимодействию.

\section{Анимация (организация обучающей жизни сети)}

Сотрудники, которые присоединяются к обучающей сети, зачастую находятся в поиске новых форм взаимодействия. Они ищут, прежде всего, нейтральную зону, в которой обмен мнениями может происходить благожелательно и правдиво. При этом нужно, чтобы этот обмен мнениями был одновременно и допустимым, и координированным. Обучающая сеть является местом для обсуждения, обмена мнениями, опытом, наблюдения других и самонаблюдения в ситуации взаимодействия. Все это служит основой для организации жизни сети (ее анимации), проявляя уважение к каждому ее члену. Таким образом, анимация является структурообразующей составляющей обучающей сети и средством формирования ее идентичности.

Помимо аниматоров, стержнем организации жизни сети является фасилитатор.

В силу их центральной роли фасилитатор обучающей сети и аниматоры каждой из ее групп выполняют следующие функции:

- организация поэтапной работы над решаемым вопросом;

- распределение времени, отведенного для каждого этапа;

- подбор методов работы в зависимости от состава участников;

- регулирование в случае возникновения трений;

- определение главной темы сессий и ритма, необходимого для мобилизации участников;

- создание банка данных для капитализации того, что было предложено участниками;

- наблюдение и контроль над процессом производства подлежащего к сдаче продукта.

Фасилитатор с аниматорами одновременно руководят развитием и хорошим функционированием обучающей сети, заботятся о создании привлекательных для участников условий и при этом следят за тем, чтобы она производила результаты, ожидаемые в первую очередь самими участниками.

\section{Какое количество участников необходимо вовлечь в обучающую сеть?}

Эмпирический анализ обучающих сетей SNCF показал, что после выработки общего видения, ра- ботая в стиле «лаборатории», менее $3 \%$ коллектива может привести к необходимым изменениям (см. рис. 1). Так, группа из 15 человек обеспечила преобразование Управления недвижимостью (на период реализации проекта в штате числилось 500 человек). Обучающая сеть «Alsace 2020», действующая в подразделении SNCF с 5 тыс. сотрудников в регионе Эльзас, начитывала 120 человек на момент ее запуска.

При условии, что руководство позволит ему работать нестандартными методами (вне нормы) и защитит его от управленческого менеджмента «указание (контроль)», коллектив обучающей сети будет экспериментировать с новым подходом, новыми услугами (например, поднос багажа на вокзале)... Во время этого эксперимента другая часть сотрудников будет учиться, и в определенный момент будет достигнут критический уровень, необходимый для преобразования.

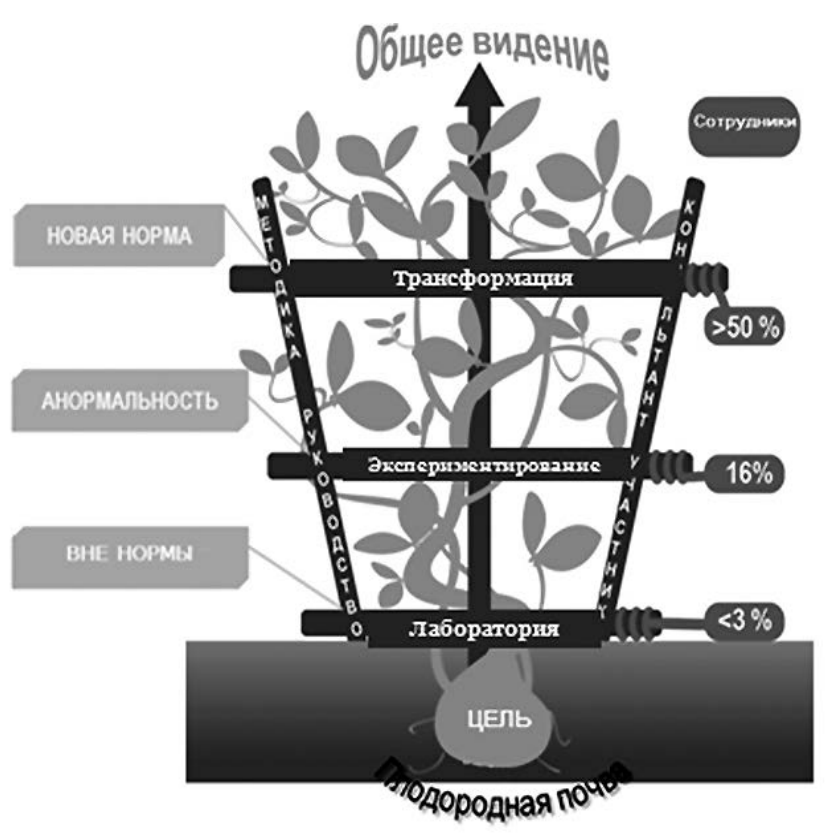

Рис. 1. Дерево транссрормации [4, с. 111]

\section{Что производит обучающая сеть?}

Основными плодами работы обучающей сети являются:

\section{Диагностика}

Диагностика состоит в приведении конкретных цифр и объективных элементов по ситуации, которая вызывает проблему или по которой возникают вопросы. Диагностики, как правило, дополняются анализами, объясняющими причины и следствия, а также рекомендациями. 
Некоторые диагностики могут включать элементы бенчмаркинга, т.е. поиска новых и более совершенных приемов работы, с использованием передового опыта других организаций.

\section{Обмен практиками}

Участники обмениваются практиками и своим опытом по рассматриваемому вопросу, описывая и логически объясняя его. При этом они не только рассказывают о своей практике, но и анализируют ее - сами по себе и (или) путем опроса других участников.

\section{Предварительное изучение}

Когда тема, над которой работает обучающая сеть, недостаточно сформирована, очень трудно сразу разработать план действий - необходима предварительная фаза ее изучения. Речь идет о тщательном рассмотрении темы с опорой на опыт, внешние и внутренние источники, на предмет выделения периметра дальнейшего обсуждения, чтобы впоследствии осуществить диагностику и наметить план действий.

\section{План действий}

Одним из основных результатов работы обучающей сети является разработка плана действий. Этот план разрабатывается с привлечением самих действующих лиц и заинтересованных сторон, с учетом стадий его внедрения, необходимых ресур- сов и показателей результативности. Отметим, что обучающая сеть представляет собой особую форму апробации проводимых изменений в действии и является, таким образом, одним из эффективных инструментов внедрения изменений.

Все вышеперечисленное может быть реализовано как отдельно, так и совместно.

Важно, особенно в начале существования обучающей сети, чтобы активно афишировались результаты, полученные с ее помощью.

Не менее важно помнить и то, что опыт, полученный участниками обучающей сети в процессе ее работы, так же важен, как и выработанные ими предложения или план действий.

\section{Последовательность стадий обучающей сети}

Процесс обучающей сети состоит из трех этапов:

- создание: Определение того, кто будет участниками обучающей сети, какие методы будут использованы для ее анимации и выбора тем для обсуждения;

- взаимодействие: Организация и поддержка взаимодействия между участниками и создание благоприятного климата для развития коллективного интеллекта и обучения;

- вклад: Индивидуальное и коллективное обучение, разработка решений и их внедрение в производ-

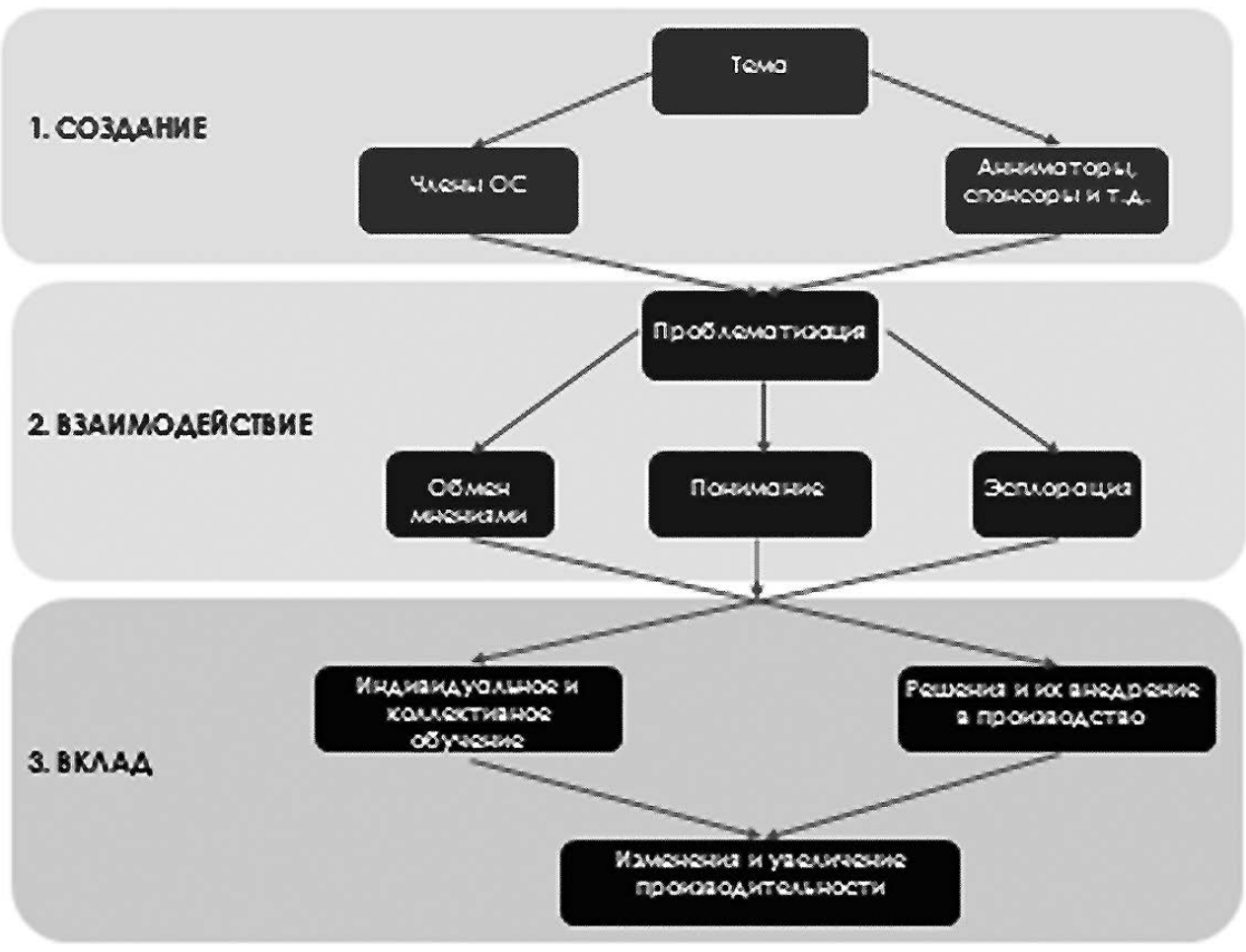

Рис. 2. Процесс развития обучающей сети [4, с. 99] 
ство. Реализация изменений и увеличение производительности.

Обучающая сеть может быть представлена следующей процедурной моделью, иллюстрирующей ее фазы и логику (см. рис. 2).

Несколько фактов из опыта использования обучающих сетей в компании SNCF

Обучающие сети используются в компании SNCF начиная с 2007 г. С этого времени в общей сложности в компании было мобилизовано порядка 30 обучающих сетей, с привлечением в них приблизительно трех тысяч сотрудников и около 300 менеджеров. Несколько тысяч сотрудников были затронуты результатами и прониклись «духом» обучающих сетей.

Обучающая сеть преследует двоякую цель:

- совместное обучение в процессе деятельности;

- создание локальных условий для возникновения и реализации инноваций в рамках общей стратегии компании.

Решения, которые вырабатываются с помощью обучающих сетей, могут быть не самыми лучшими, но они полезны, удобны и используются, так как являются решениями тех, кого они непосредственно касаются.

Обучающие сети вносят свою лепту в достижение настоящих скачков производительности и способствуют личностному развитию своих участников.

В частности, обучающие сети обеспечивают:

- повышение производственных показателей;

- увеличение организационной вовлеченности;

- чувство принадлежности к компании;

- признание заслуг;

- осмысленность деятельности;

- повышение доверия;

- взаимодействие между различными иерархическими уровнями.

Ниже приведены примеры результатов, достигнутых с помощью обучающих сетей.

Дирекция закупок SNCF:

- 118 млн евро экономии вместо плановых 100 млн евро;

- оценка удовлетворенности клиентов составила 8/10 при целевом показателе 7/10.

\section{TER Нормандии:}

- $96 \%$ соблюдения расписания;

- уменьшение количества отмененных поездов по причине технических неполадок;

- улучшение информированности клиентов благодаря налаженному контакту между различными специалистами;
- уменьшение количества внештатных ситуаций благодаря оказанию помощи пассажирам с ограниченной подвижностью;

- ввод новых услуг для клиентов (перенос багажа на вокзале, совместные автоперевозки и т.д.).

TGV Est:

- увеличение индекса удовлетворенности клиентов информацией, предоставляемой в поезде в случае внештатных ситуаций, с $52 \%$ до $60 \%$ менее чем за 2 года;

- создание диспетчерских центров по всем веткам TGV - от централизации к локализации.

Vigirail:

Предложение и разработка нового программного обеспечения для технического обслуживания в рамках программы Vigirail. Данная программа была введена в действие Французским обществом железных дорог (SNCF) в октябре 2013 г. в целях усиления безопасности перевода стрелок и модернизации технического обслуживания.

\section{Преимущества и условия для успеха обучающей сети}

Здесь перечислены преимущества обучающей сети и условия для ее успеха, по свидетельствам членов обучающей сети Management et $L, A, J$ (отделения Transilien, парижского региона Сен-Лазар) [4, c. 52-54].

\section{Преимущества:}

- возможность участников обучающей сети проявлять инициативу;

- способность обучающей сети устранять препятствия и барьеры для улучшения функционирования организации;

- содействие обучающей сети углублению знаний о компании, трансверсальному функционированию и укреплению связей между профессиями;

- способность обучающей сети производить результаты.

Необходимые условия для продуктивного функционирования обучающей сети:

- сохранять внеиерархичный характер рабочих групп несмотря на трудности;

- афишировать результаты, полученные в результате работы групп обучающей сети, с целью ее легитимизации и усиления производимых ею эффектов;

- позиционирование руководителей как помощников, а не в качестве тех, кто принуждает и ограничивает. 


\section{Заключение}

Обучающиеся сети являются одним из инструментов в распоряжении организаций компаний и предприятий, который позволяет успешно внедрять изменения и управлять ими для эффективного адаптирования к сложностям постоянно меняющегося мира.

По своей природе обучающиеся сети способствуют повышению качества жизни на работе благодаря взаимодействиям, которые они обеспечивают, и инновациям, которые они производят. Они содействуют развитию личностному и коллективному, благоприятствуют формированию у каждого участника видения личного смысла его работы, признанию его идей и благодарности за его работу. Все это позволяет участникам «заглядывать вперед» и, таким образом, лучше воспринимать преобразования и изменения.

\section{Литература}

1. Argyris C., Schön D.-A. Organizational Learning II, Reading, Mass: Addison Wesley, 1996.

2. Argyris C., Schön D. Apprentissage organisationnel: théorie, méthode, pratique, Bruxelles, De Boeck Supérieur. Management, 2001.

3. Autissier D., Giraud L., Jonson K.J., Moutot J.-M. Les liens entre les caractéristiques et le succès des changements organisationnels: le cas d'une start-up // Question(s) de Management?, $\mathrm{No}^{\circ} 3$, septembre 2013, pp. 11-24.

4. Autissier D., Hureau J.-P., Raynard T., VandangeonDerumez $I$. Les réseaux apprenants : Une démarche d'accompagnement du changement dans la relation de service à la SNCF. Paris, Eyrolles, 2013.

5. Bercovitz J., Feldman M. Academic entrepreneurs: Organisational change and the individual level // Organization Science, 2008, 19(1), pp.69-89.

6. Landier $H$. Vers l'entreprise intelligente. Paris, CalmannLevy, 1991.

7. Lüscher L., Lewis M. Organizational change and managerial sensemaking: Working through paradox // Academy of Management Journal, 2008, 51 (2), pp. 221-240.

8. Morin E. La méthode (6 tomes). Paris, Editions du Seuil, 1977 (t. 1).

9. Morin E. Introduction à la pensée complexe. Paris, Editions du Seuil, 2005

10. PROFIL.SNCF [Electronic resource] // www.sncf.com/ ressources/reports/sncf_profil_2015.pdf(дата обращения: 09.11.2015).

11. Senge P.M. The Fifth Discipline. New York, Doubleday, 1990.

12. Senge $P$. La Cinquième Discipline. L'art et la manière des organisations qui apprennent. Paris, First Editions, 1991.

13. Senge $P$. La Cinquième Discipline. Levier des organisations apprenantes. Eyrolles, Paris, 2015.

14. SOL France [Electronic resource] // www.solfrance.org (дата обращения 29.03.2014).

15. Zarifian P. Objectif compétence. Paris, Editions Liaisons, 1999.

\section{Refereces}

1. Argyris C., Schön D.-A, Organizational Learning II, Reading, Mass: Addison Wesley, 1996.

2. Argyris C., Schön D., Apprentissage organisationnel : théorie, méthode, pratique, Bruxelles, De Boeck Supérieur. Management, 2001.

3. Autissier D., Giraud L., Jonson K.J., Moutot J.-M. Les liens entre les caractéristiques et le succès des changements organisationnels : le cas d'une start-up. Question(s) de Management?, $\mathrm{N}^{\circ} 3$, septembre 2013, pp. 11-24.

4. Autissier D., Hureau J.-P., Raynard T., Vandangeon-Derumez $I$. Les réseaux apprenants: Une démarche d'accompagnement du changement dans la relation de service à la SNCF. Paris, Eyrolles, 2013.

5. Bercovitz J., Feldman, M. Academic entrepreneurs : Organisational change and the individual level. Organization Science, 2008, 19(1), pp.69-89.

6. Landier $H$. Vers l'entreprise intelligente. Paris, CalmannLevy, 1991.

7. Lüscher L., Lewis M. Organizational change and managerial sensemaking: Working through paradox. Academy of Management Journal, 2008, 51 (2) pp. 221-240.

8. Morin E., La méthode (6 tomes). Paris, Editions du Seuil, 1977 (t.1).

9. Morin E., Introduction à la pensée complexe. Paris, Editions du Seuil, 2005

10. PROFIL.SNCF. Available at: www.sncf.com/ressources/ reports/sncf_profil_2015.pdf (accessed 9 November 2015).

11. Senge P.M. The Fifth Discipline. New York, Doubleday, 1990.

12. Senge $P$., La Cinquième Discipline. L'art et la manière des organisations qui apprennent. Paris, First Editions, 1991.

13. Senge P., La Cinquième Discipline. Levier des organisations apprenantes. Eyrolles, Paris, 2015.

14. SOL France. Available at: www.solfrance.org (accessed 29 March 2014).

15. Zarifian P., Objectif compétence. Paris, Editions Liaisons, 1999. 